\title{
Type 1 regulatory $T$ (Tr1) cells: from the bench to the bedside
}

\author{
Silvia Gregori ${ }^{1 *}$, Rosa Bacchetta ${ }^{1}$, Manuela Battaglia ${ }^{2}$, Maria Grazia Roncarolo ${ }^{1,3}$ \\ From 7th European Workshop on Immune-Mediated Inflammatory Diseases \\ Noordwijk aan Zee, the Netherlands. 28-30 November 2012
}

\section{Background}

Type 1 regulatory $\mathrm{T}(\operatorname{Tr} 1)$ cells are an inducible subset of regulatory $\mathrm{T}$ cells that play a pivotal role in promoting and maintaining tolerance. The main mechanisms by which $\operatorname{Tr} 1$ cells control immune responses are the secretion of high levels of IL-10, and the killing of myeloid myeloid cells through the release of Granzyme B. To date a defined cell surface signature has not been identified for Tr1 cells and their characterization has thus relied on their unique cytokine production profile. Tr1 cells secrete high levels of IL-10 and minimal amounts of IL-4 and IL-17, which distinguish them from Th2 and Th17 [1]. Furthermore, Tr1 cells secrete low levels of IL-2, and depending on the local cytokine milieu can produce variable levels of IFN- $\gamma$. Similar to other $\mathrm{T}$ cell subsets, $\mathrm{Tr} 1$ cells can transiently express FOXP3 upon activation; however, in Tr1 cells FOXP3 expression is not constitutive and never reaches the high levels characteristic of $\mathrm{CD} 25^{+} \mathrm{Foxp}^{+}{ }^{+}$regulatory $\mathrm{T}$ cells.

During the last decade, regulatory $\mathrm{T}$ cell-based therapies have become an attractive therapeutic option for inducing/restoring tolerance. Several protocols to generate $\operatorname{Tr} 1$ cells in vitro or to isolate Tr1 cell clones have been developed. We established a reproducible method to generate allo-specific Tr1 cells in vitro using recombinant IL-10 or IL-10-derived from tolerogenic dendritic cells (DC).

\section{Results}

Tr1 cells are induced in vitro using a new subset of human tolerogenic dendritic cells (DC), termed DC-10, which are present in vivo and inducible in vitro from monocytes in the presence of IL-10 [2]. Resulting T cells are anergic, secrete significant levels of IL-10, and suppress T cell responses in vitro in an IL-10-dependent manner. Adoptive transfer of ex vivo induced alloantigen-specific Tr1

'San Raffaele Telethon Institute for Gene Therapy (HSR-TIGET), Dept. of

Regenerative Medicine, Stem Cells, and Gene Therapy, Milan, Italy

Full list of author information is available at the end of the article cells has proven to be feasible and safe, and can be applied in allogeneic hematopoietic stem cell transplantation. An alternative strategy for the induction of high numbers of human Tr1 cells is lentiviral-mediated gene transfer of human IL-10. Stable ectopic expression of IL-10 can efficiently generate homogeneous populations of $\operatorname{Tr} 1$-like cells [3]. These cells display potent suppressive functions both in vitro and in vivo in xenogeneic graft versus host disease model, while preserving the graft versus leukemia effects.

\section{Conclusions}

Tr1 cells can be induced in vitro for Tr1-based cell therapy aimed at restoring peripheral tolerance in immunemediated diseases.

\section{Author details}

'San Raffaele Telethon Institute for Gene Therapy (HSR-TIGET), Dept. of Regenerative Medicine, Stem Cells, and Gene Therapy, Milan, Italy. ${ }^{2}$ San Raffaele Diabetes Institute (OSR-DRI), Dept. of Immunology, Milan, Italy. ${ }^{3}$ Vita-Salute San Raffaele University, Milan, Italy.

\section{Published: 28 November 2012}

\section{References}

1. Gregori S, Goudy KS, Roncarolo MG: The cellular and molecular mechanisms of immuno-suppression by human type 1 regulatory T cells. Front Immunol 2012, 3:30, Epub 2012 Feb 29.

2. Gregori S, Tomasoni D, Pacciani V, Scirpoli M, Battaglia M, Magnani CF, Hauben $\mathrm{E}$, Roncarolo MG: Differentiation of type $1 \mathrm{~T}$ regulatory cells (Tr1) by tolerogenic DC-10 requires the IL-10-dependent ILT4/HLA-G pathway. Blood 2010, 116(6):935-44, Epub 2010 May 6. Erratum in: Blood. 2011 Nov 3;118(18):5060.

3. Andolfi G, Fousteri G, Rossetti M, Magnani CF, Jofra T, Locafaro G, Bondanza A, Gregori S, Roncarolo MG: Enforced IL-10 expression confers Type 1 Regulatory T cell (Tr1) phenotype and function to human CD4(+) T cells. Mol Ther 2012, 20(9):1778-90, doi: 10.1038/mt.2012.71. Epub 2012 Jun 12.

doi:10.1186/1479-5876-10-S3-17

Cite this article as: Gregori et al:: Type 1 regulatory T (Tr1) cells: from the bench to the bedside. Journal of Translational Medicine 2012 10(Suppl 3):17. 